\title{
Testing the comet nature of main belt comets. The spectra of 133P/Elst-Pizarro and 176P/LINEAR
}

\author{
J. Licandro ${ }^{1,2}$, H. Campins ${ }^{3}$, G. P. Tozzi ${ }^{4}$, J. de León ${ }^{5}$, N. Pinilla-Alonso ${ }^{6}$, H. Boehnhardt ${ }^{7}$, and O. R. Hainaut ${ }^{8}$ \\ ${ }^{1}$ Instituto de Astrofísica de Canarias, c/Vía Láctea s/n, 38200 La Laguna, Tenerife, Spain \\ e-mail: jlicandr@iac.es \\ 2 Departamento de Astrofísica, Universidad de La Laguna, 38205 La Laguna, Tenerife, Spain \\ 3 Physics Department, University of Central Florida, Orlando, FL 32816, USA \\ 4 INAF - Osservatorio Astrofisico di Arcetri, 50125 Firenze, Italy \\ 5 Instituto de Astrofísica de Andalucía, Granada, Spain \\ 6 NASA Postdoctoral Program at NASA Ames Research Center, Moffett Field, CA, USA \\ 7 Max-Planck Institute for Solar System Research, 37191 Katlenburg-Lindau, Germany \\ ${ }^{8}$ ESO, Karl Schwarzschild Straße, 85748 Garching bei München, Germany
}

Received 4 April 2011 / Accepted 7 June 2011

\begin{abstract}
Context. Dynamically, 133P/Elst-Pizarro and 176P/LINEAR are main belt asteroids, likely members of the Themis collisional family, and unlikely of cometary origin. They have been observed with cometary-like tails, which may be produced by water-ice sublimation. They are part of a small group of objects called Main Belt Comets (MBCs, Hsieh \& Jewitt 2006).

Aims. We attempt to determine if these MBCs have spectral properties compatible with those of comet nuclei or with other Themis family asteroids.

Methods. We present the visible spectrum of MBCs 133P and 176P, as well as three Themis family asteroids: (62) Erato, (379) Huenna and (383) Janina, obtained in 2007 using three telescopes at "El Roque de los Muchachos" Observatory, in La Palma, Spain, and the $8 \mathrm{~m}$ Kueyen (UT2) VLT telescope at Cerro Paranal, Chile. The spectra of the MBCs are compared with those of the Themis family asteroids, comets, likely "dormant" comets and asteroids with past cometary-like activity in the near-Earth (NEA) population. As 133P was observed active, we also look for the prominent $\mathrm{CN}$ emission around $0.38 \mu$ m typically observed in comets, to test if the activity is produced by the sublimation of volatiles.

Results. The spectra of $133 \mathrm{P}$ and 176P resemble best those of B-type asteroid and are very similar to those of Themis family members and another activated asteroid in the near-Earth asteroid population, (3200) Phaethon. On the other hand, these spectra are significantly different from the spectrum of comet 162P/Siding-Spring and most of the observed cometary nuclei. $\mathrm{CN}$ gas emission is not detected in the spectrum of 133P. We determine an upper limit for the $\mathrm{CN}$ production rate $Q(\mathrm{CN})=1.3 \times 10^{21} \mathrm{~mol} / \mathrm{s}$, three orders of magnitude lower than the $Q(\mathrm{CN})$ of Jupiter family comets observed at similar heliocentric distances.

Conclusions. The spectra of 133P/Elst-Pizarro and 176P/LINEAR confirm that they are likely members of the Themis family of asteroids, fragments that probably retained volatiles, and unlikely have a cometary origin in the trans-Neptunian belt or the Oort Cloud. They have similar surface properties to activated asteroids in the NEA population, which supports the hypothesis that these NEAs are scattered MBCs. The low $Q(\mathrm{CN})$ of $133 \mathrm{P}$ means that, if water-ice sublimation is the activation mechanism, the gas production rate is very low and/or the parent molecules of $\mathrm{CN}$ present in the nuclei of normal comets are much less abundant in this MBC.
\end{abstract}

Key words. minor planets, asteroids: individual: 133P/Elst-Pizarro - minor planets, asteroids: individual: 176P/LINEAR minor planets, asteroids: general - comets: general - techniques: spectroscopic

\section{Introduction}

Comets are known to originate in the trans-Neptunian belt (TNB) and the Oort Cloud. Observationally, comets are distinguished from asteroids by the presence of a coma and/or tail. However, this is not a conclusive criterion. E.g. icy objects only develop a coma if the temperature is sufficient to sublimate ices, so distant comets may not show activity.

For the purpose of this paper we define that an object has a cometary origin if it shows similar physical properties to objects that have been considered comets up to now, i.e., icy objects scattered from the TNB or the Oort Cloud.

The Tisserand parameter with respect to Jupiter $\left(T_{\mathrm{J}}\right)$ provides a simple way to discriminate dynamically between asteroids and comets (Kresak 1982; Kosai 1992). Main-belt asteroids move in orbits with $T_{\mathrm{J}}>3$, while comets have unstable orbits with $T_{\mathrm{J}}<3$.

Recently, some objects in orbits indistinguishable from those of other main belt asteroids have been observed "active" (with a dust coma and/or tail). They have been called main-belt comets (MBCs) (Hsieh \& Jewitt 2006). 133P/Elst-Pizarro (hereafter, 133P) and 176P/LINEAR (hereafter, 176P) are also identified as asteroids 7968 and 118401, respectively. They have $T_{\mathrm{J}}=3.184$ and 3.166, respectively, and they are two of the seven objects observed so far that can be considered as MBCs following Hsieh \& Jewitt (2006) pure phenomenogical definition.

Dynamical simulations show that MBCs are extremely unlikely to originate in the TNB or the Oort Cloud (e.g., Fernández et al. 2002), indicating that they likely formed in situ. Recent 
work suggests that some icy trans-Neptunian objects (TNOs) might have been delivered to the asteroid belt during the late heavy bombardment (Levison et al. 2009), but even those simulations fail to produce low inclination, low-eccentricity orbits such as those of $133 \mathrm{P}$ and $176 \mathrm{P}$.

$133 \mathrm{P}$ is the first discovered MBC (Elst et al. 1996) and, so far, the best characterized one. Since then, it has been observed active every perihelion passage around the orbital quadrant following perihelion, and inactive the two quadrants around aphelion (Boehnardt et al. 1997; Toth 2006; Hsieh et al. 2006, 2009, 1010; Bagnulo et al. 2010). This recurrent activity supports the hypothesis that $133 \mathrm{P}$ has an ice reservoir, which periodically sublimates and elevates dusty material into the coma and tail region. The dust coma and tail, and not a gas emission feature, are so far the only signatures of ongoing activity in MBCs. On the other hand, 176P is the third known MBC and it exhibited cometary activity only at the time of its discovery in 2005 (Hsieh et al. 2006). It was never reported active since.

Understanding the origin of these bodies is crucial. If they are formed "in situ" and, in particular, if they are members of a collisional family and if their activity is due to water ice sublimation, there should be water ice in many asteroids. If they are captured TNB or Oort Cloud comets, the mechanisms that drove them to their present orbits needs to be understood. The existence of ice in main-belt objects is surprising given their proximity to the Sun, and presents intriguing opportunities for constraining the temperature, composition and structure of primitive asteroids and our protoplanetary disk. Recent theoretical model calculations indicate that subsurface ice can survive in an MBC over the lifetime of the solar system (Schorghofer 2008).

The $R$-band albedo of $133 \mathrm{P}$ and $176 \mathrm{P}$ is $p_{R}=0.05 \pm 0.02$ and $p_{R}=0.06 \pm 0.02$, respectively (Hsieh et al. 2009). The $133 \mathrm{P}$ albedo determination is in agreement with more recent estimates by Bagnulo et al. (2010), using the polarization vs. phase function and assuming that the relation with the albedo is the same of that found for asteroids $\left(p_{R}=0.07 \pm 0.01\right)$. All these values are consistent with the albedos of primitive C-, B-, D-type asteroids and cometary nuclei (Fernández et al. 2005), implying that the albedo does not appear to be a decisive diagnostic parameter for determining the source zone of MBCs.

The orbits of 133P and 176P are typical of Themis collision family members, or a sub-family of it, called the Beagle family (Nesvorny et al. 2008). The Themis family is likely the result of the breakup of a parent asteroid about $370 \mathrm{~km}$ in diameter $\sim 1$ Gyr ago (Marzari et al. 1995; Tanga et al. 1999; Nesvorny et al. 2008) and is one of the largest and statistically most robust asteroid families (e.g., Carusi \& Valsecchi 1982; Zappalà et al. 1990). The Beagle family is younger than the Themis one, with an age $<10$ My (Nesvorny et al. 2008). The surface of 133P and $176 \mathrm{P}$ is neutral in color (Hammergren 1996; Hsieh et al. 1010; Bagnulo et al. 2010), consistent with the nearly neutral reflection spectra of primitive asteroids of the C-complex, but bluer than the colors of the majority of cometary nuclei (Lamy \& Toth 2009). Given their origin from a common parent, Themis family members are thought to be compositionally homogeneous, as corroborated by spectroscopical studies showing that the family is dominated by primitive C-type asteroids that also exhibit signs of aqueous alteration (Bell 1989; Florczak et al. 1999; Ivezic et al. 2002; Mothé-Diniz et al. 2005). The detection of water ice on the surface of the largest member of the family (24) Themis (Campins et al. 2010; Rivkin \& Emery 2010) and the evidence of aqueous alteration in about $50 \%$ of the Themis family asteroids strongly support that water is widely present in the Themis family.
In this paper we present and study spectra of two MBCs, $133 \mathrm{P}$ and $176 \mathrm{P}$ to address the open question of the origin of this population and, in particular, to discriminate if they are Themis family asteroids or interloper comets. In Sect. 2 we present visible spectra of both MBCs obtained using different telescopes, aditionaly we present new spectra of three Themis family asteroids. In Sect. 3 we compare the spectra of the MBCs with spectra of objects with asteroidal and cometary nature: the spectra of Themis family asteroids, the spectrum of (3200) Phaethon, a prototype of near-Earth asteroid that presented cometary-like activity in the past and possibly linked to MBCs (Meng et al. 2004; Licandro et al. 2007); and the spectra of comet nuclei. In Sect. 4 we determine an upper limit for the $\mathrm{CN}$ production rate of 133P, and we present our conclusions in Sect. 5.

\section{Observations and data reduction}

Visible spectra of 133P were obtained during its 2007 perihelion passage using three telescopes: the $3.5 \mathrm{~m}$ Telescopio Nazionale Galileo (TNG) and the $4.2 \mathrm{~m}$ William Herschel Telescope (WHT) at "El Roque de los Muchachos" Observatory (ORM, Canary Islands, Spain), and the $8 \mathrm{~m}$ Kueyen (UT2) VLT telescope at Cerro Paranal (Chile). On the other hand, visible spectra of 176P were obtained on January 20, 2007 using the $2.5 \mathrm{~m}$ Nordic Optical Telescope (NOT) at the ORM.

The DOLORES spectrograph at the TNG was used on June 17, 2007 to observe 133P. Spectra using the LR-B and the LR-R grisms were obtained, with dispersions of 2.52 and $2.61 \AA /$ pixel, respectively. The $5.0^{\prime \prime}$ slit width was used, oriented at the parallactic angle to minimize the slit losses due to atmospheric dispersion. Three spectra with an exposure time of $600 \mathrm{~s}$ each of 133P where obtained with the LR-R grism, covering the $0.52-0.92 \mu \mathrm{m}$ spectral range. The object was shifted in the slit direction by $5^{\prime \prime}$ between consecutive spectra to better correct the fringing. Two spectra of $1200 \mathrm{~s}$ each of 133P where obtained with the LR-B grism, covering the 0.37 to $0.70 \mu \mathrm{m}$ spectral range.

A spectrum of $133 \mathrm{P}$ was also obtained with the WHT on Jun. 17, 2007, using the double arm ISIS spectrograph. Spectra in the red and blue arms where obtained simultaneously, using the R300B grating in the blue arm, with a dispersion of $0.86 \AA /$ pixel, and the R316R grating in the red arm, with a dispersion of $0.93 \AA /$ pixel. A $3^{\prime \prime}$ slit width was used, oriented at parallactic angle. A single $900 \mathrm{~s}$ exposure was done in both arms. 133P was observed again on July 9, 2007 with the WHT using ISIS. Spectra in the red and blue arms where obtained simultaneously, using the R300B grating in the blue arm, and the R158R grating in the red arm (with a dispersion of $1.81 \AA /$ pixel). A $5^{\prime \prime}$ slit width was used oriented at the parallactic angle. Two spectra of $1800 \mathrm{~s}$ where obtained in both arms.

Finally, 133P was observed on July 21, 2007 with the VLT using the camera spectrograph FORS2. A total of 7 lowresolution spectra of $300 \mathrm{~s}$ each were obtained. The grism 150I without an order sorting filter and a slit of $1.3^{\prime \prime}$ width were used. The covered spectral range was $0.33-0.66 \mu \mathrm{m}$ with a dispersion of $230 \AA / \mathrm{mm}$, corresponding to about $3.5 \AA /$ pixel. Solar analogue star HD 209847 was also observed in order to obtain the reflectance spectrum. The observations were done in service mode, and all the spectra were recorded at airmass $>1.6$. Since the slit was not oriented in the parallactic angle, the spectra suffered from strong atmospheric refraction and they could not be used to measure the spectral reflectance. But the signal-to-noise ratio (SNR) of the obtained spectrum was sufficiently hight to 
search for the presence of gas emission, as that of CN at $3880 \AA$ (see Sect. 4). To do that, the spectrum of spectrophotometric standard LTT 377 was obtained for calibration purposes using the same configuration.

On January 20.22 UT, 2007, 176P was observed with the $2.5 \mathrm{~m}$ NOT telescope using the camera-spectrograph ALFOSC. The 5.0" slit width was used, oriented in the parallactic angle. Two spectra of $1800 \mathrm{~s}$ were obtained with the \#4 grism, covering the $0.32-0.91 \mu \mathrm{m}$ spectral range, with a dispersion of $3 \AA /$ pixel.

Images were overscan and bias corrected, and flat-field corrected using lamp flats. The two-dimensional spectra were extracted, sky background subtracted, and collapsed to one dimension. The wavelength calibration was done using the Neon and Argon lamps. The spectra of each asteroid, obtained at different positions in the slit, were averaged.

To correct for telluric absorption and to obtain the relative reflectance, a G star from the Landolt list (Landolt 1992) was observed at different airmases (similar to those of the object) before and after the asteroid's observations, and used as a solar analogue star. Landolt (SA) 115-271, and Landolt (SA) 107-684 were observed to correct the spectra of $133 \mathrm{P}$ and 176P, respectively. The spectrum of each solar system object was divided by that of the corresponding solar analogue star, and then normalized to unity around $0.55 \mu \mathrm{m}$, thus obtaining the normalized reflectance. The obtained spectra of $133 \mathrm{P}$ are shown in Fig. 1. The spectrum of $176 \mathrm{P}$ is shown in Fig. 2 together with the weighted mean spectrum of the 3 spectra of 133P shown in Fig. 1. In Fig. 2 both spectra are rebinned to a resolution of $30 \AA$. The spectrum of $176 \mathrm{P}$ is plotted only in the $0.4-0.72 \mu \mathrm{m}$ region because of the very low SNR at bluer wavelengths and the strong fringing in the red.

Also asteroids (62) Erato, (379) Huenna and (383) Janina, all of them considered to be members of the Themis family, were observed on April 1, 2006 with the WHT using the ISIS spectrograph. Spectra in the red and blue arms were obtained simultaneously, using the R300B and R158R gratings, respectively. A $5^{\prime \prime}$ slit was used oriented in the parallactic angle. Solar analogue star BS 4486 and G-type Landolt star SA 107-689 were observed to obtain the relative reflectance of the asteroids. The resulting reflectance spectra, processed in a similar way as those of $133 \mathrm{P}$ and 176P, are shown in Fig. 3. The spectra of these Themis family asteroids are used to compare with that of $133 \mathrm{P}$ and $176 \mathrm{P}$.

Notice that (62) Erato, (379) Huenna and (383) Janina, with diameter $95.4 \pm 2.0,92.3 \pm 1.7$ and $45.5 \pm 1.8 \mathrm{~km}$, respectively (Tedesco et al. 2004), are larger than 133P and 176P (diameter $3.8 \pm 0.6$ and $4.0 \pm 0.4 \mathrm{~km}$, respectively, Hsieh et al. 2009). We observed these Themis family asteroids because small objects like $133 \mathrm{P}$ and $176 \mathrm{P}$ are very faint and need much longer exposure times to obtain similar $\mathrm{S} / \mathrm{N}$.

\section{Data analysis}

The spectra of $133 \mathrm{P}$ and $176 \mathrm{P}$ are very similar, presenting a neutral to slightly bluish slope at wavelengths larger than $5000 \AA$, and a drop in the UV region. This behavior is typical of B-type asteroids, according to the Tholen spectrophotometric classification system (Tholen 1989). Measuring the spectral slope in the visible region $\left(S_{\mathrm{V}}^{\prime}\right)$ as in Licandro et al. (2008), we obtain $S_{\mathrm{V}}^{\prime}=$ $0 \pm 1 \% / 1000 \AA$ for $133 \mathrm{P}$ and $S_{\mathrm{V}}^{\prime}=-3 \pm 1 \% / 1000 \AA$ for $176 \mathrm{P}$. $176 \mathrm{P}$ is spectroscopically a B-type asteroid, while the neutral slope of $133 \mathrm{P}$ places it on the line between B- or C-type asteroids. B-, C- and F-type asteroids belong to the C-complex, which

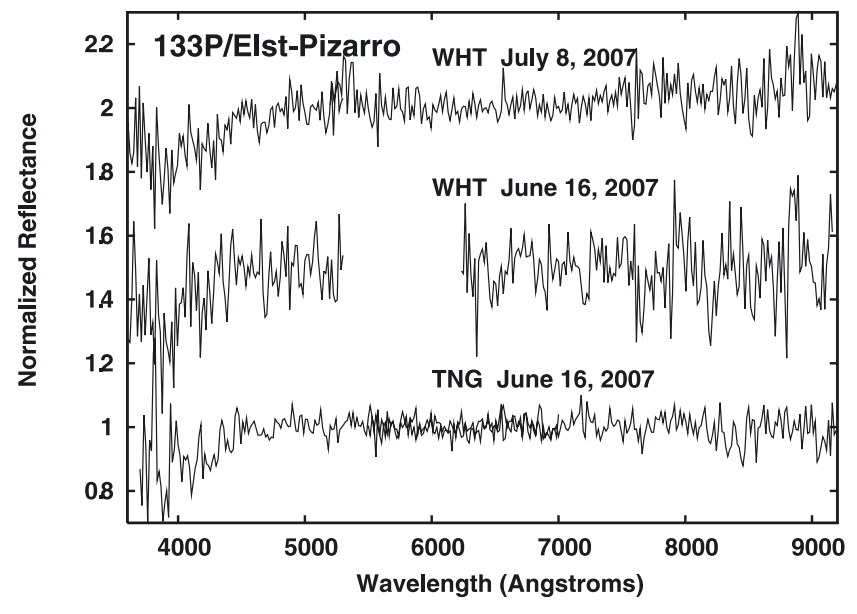

Fig. 1. Spectra of 133P/Elst-Pizarro obtained with the TNG and WHT telescopes. Spectra are normalized to unity at $5500 \AA$ and shifted vertically by 0.3 for the sake of clarity. The spectrum obtained on June 16 with the WHT (middle one) does not cover the whole spectral range (there is a "hole" between 5400 and $6200 \AA$ ), in this case both parts (red and blue arm) are scaled using TNG spectrum obtained in the same night.

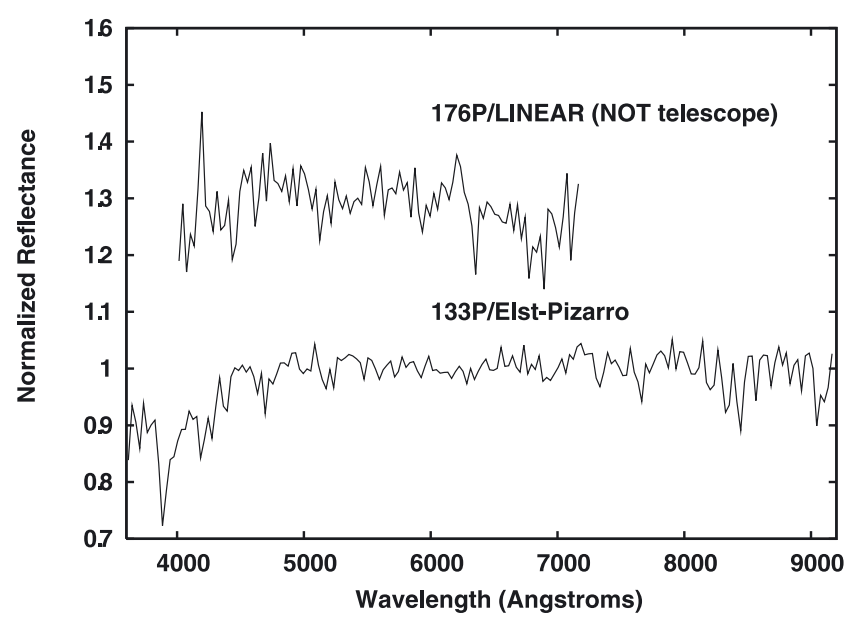

Fig. 2. Spectrum of 176P/LINEAR obtained with the NOT telescope binned to a resolution of $30 \AA$, compared with the mean spectrum of 133P/Elst-Pizarro also binned to the same resolution. Spectra are normalized to unity at $5500 \AA$ and shifted vertically by 0.3 for clarity. The spectrum of $176 \mathrm{P}$ is plotted only in the 4000-7200 $\AA$ range as the SNR outside this spectral region is very low. Notice that the spectra of $133 \mathrm{P}$ and $176 \mathrm{P}$ are similar, both show the slightly and rare negative (blue) slope in this wavelength region, typical of B-type asteroid.

comprises asteroids with a primitive composition and very low albedo, usually linked to the CI, CM carbonaceous chondrites.

Bagnulo et al. (2010), based on photometric and polarimetric measurements concluded that the overall light scattering behavior (photometry and polarimetry) of $133 \mathrm{P}$ is different from that of cometary nuclei and resembles most closely that of F-type asteroids. However, the most distinctive spectral feature of the F-type asteroids, as compared to other types, is the absence of an UV absorption (Tholen 1989) like that observed in the spectra of $133 \mathrm{P}$ and $176 \mathrm{P}$.

Recently, Clark et al. (2010) and de León et al. (2011) analyzed visible and near infrared spectra (covering the 0.5 to $2.5 \mu \mathrm{m}$ region) of asteroids classified as B-type from their visible spectra. Both papers point out that the spectra of B-type asteroids 


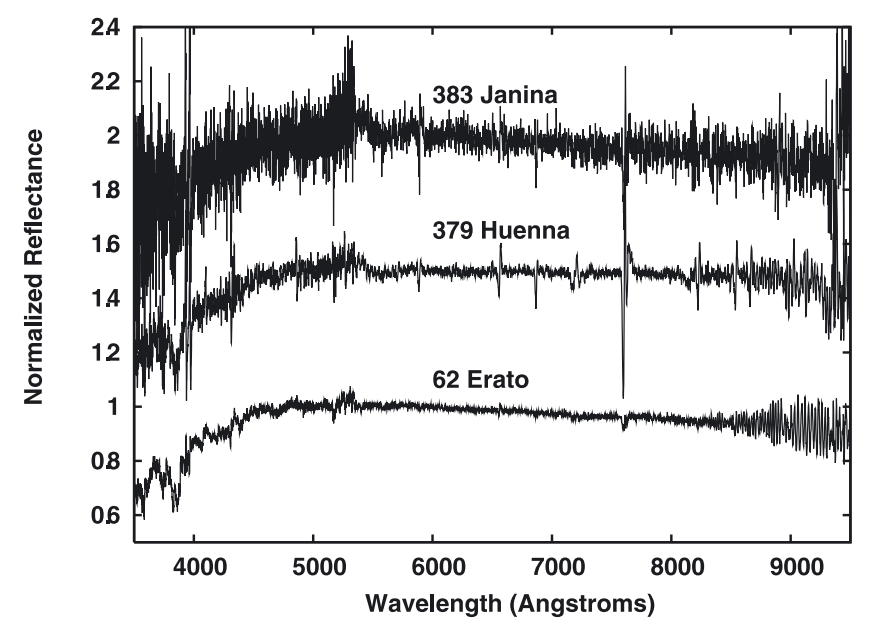

Fig. 3. Spectra of 3 Themis family asteroids obtained with the WHT. Spectra are normalized to unity at $5500 \AA$ and shifted vertically by 0.5 . Notice they are all very similar.

show a range of behaviors in the infrared region. Hence, B-type asteroids can have different light scattering properties and some of these asteroids, as could be the case of 133P (Bagnulo et al. 2010), can have photometric and polarimetric properties that resemble most closely those of F-type asteroids.

\subsection{MBCs and the Themis family}

As mentioned in the introduction, asteroids $133 \mathrm{P}$ and $176 \mathrm{P}$ can show cometary-like tails. According to their dynamical properties, they also belong to the Themis family (Haghighipour 2009). Therefore, we first compare their spectra with the spectra of other Themis family asteroids to see if these MBCs are compatible with being fragments produced by the collision that produced the family.

The Themis family is compositionally primitive, and the taxonomy of its family members is very homogeneous. Themis family asteroids are mainly $\mathrm{C}$ - and B-types in the Tholen classification system (e.g., Mothe-Diniz et al. 2005; Florczak et al. 1999). Considering Bus taxonomy (Bus 1999), there are 36 asteroids belonging to the $\mathrm{C}$ complex (6 C-type, 17 B-type, 5 Ch-type, and $8 \mathrm{Cb}$-type) and 7 asteroids to the $\mathrm{X}$ complex (5 X-type, $1 \mathrm{Xc}$-type, and $1 \mathrm{Xk}$-type). This is compatible with the breakup of a large $(\sim 370 \mathrm{~km}$ according to Tanga et al. 1999) C-class parent body. The spectra of the three Themis family asteroids presented here (62 Erato, 379 Huenna and 383 Janina) are very similar, typical of B-type asteroids. In Fig. 4 we compare the spectrum of $133 \mathrm{P}$ with that of (62) Erato, which is the one with the best SNR. Notice that the spectra of 133P and (62) Erato are very similar within the uncertainties, supporting that $133 \mathrm{P}$ and $176 \mathrm{P}$ are likely fragments of the Themis family parent body. The spectrum of (62) Erato is slightly bluer $\left(S_{\mathrm{V}}^{\prime}=\right.$ $-2 \pm 1 \% / 1000 \AA)$ than that of $133 \mathrm{P}\left(S_{\mathrm{V}}^{\prime}=0 \pm 1 \% / 1000 \AA\right)$, but similar within the errors, and both have a pronounced absorption feature in the UV. To quantify this absorption feature we compute the ratio $(\tau)$ between the reflectance at $0.4 \mu \mathrm{m}$ and the continuum at the same wavelenght computed by a linear fitting of the reflectance between 0.5 and $0.7 \mu \mathrm{m} . \tau=0.89$ and 0.83 for $133 \mathrm{P}$ and Erato, respectively.

In addition, Rousselot et al. (2011) present a near-infrared spectrum of $133 \mathrm{P}$ in the $1.1-2.4 \mu \mathrm{m}$ region that it is compatible with those of Themis-like B-types in Clark et al. (2010).

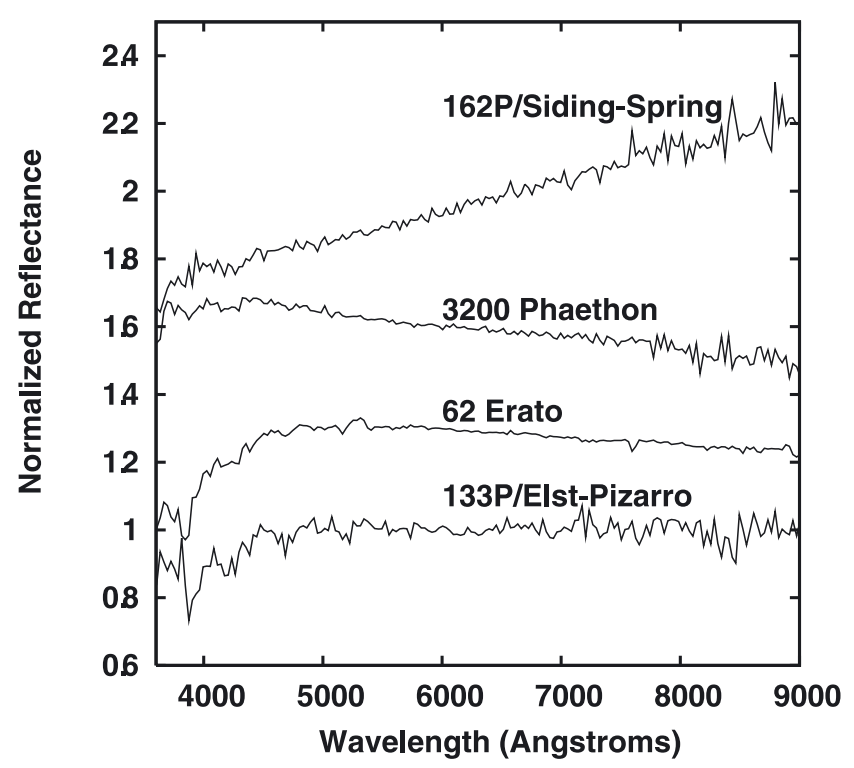

Fig. 4. Visible spectra of (62) Erato, (3200) Pahethon, 162P/SidingSpring and $133 \mathrm{P} /$ Elst-Pizarro. The spectra are normalized to unity at $5500 \AA$ and the first three are shifted vertically for clarity.

\subsection{MBCs and activated asteroids in the near-Earth objects (NEO) population}

MBCs are not the only objects in asteroidal orbits that present cometary-like activity. There is a group of asteroids in the near-Earth population with associated meteor showers: (3200) Phaethon, $2001 \mathrm{YB}_{5}$ and 2005 UD (Whipple 1983; Meng et al. 2004; and Ohtsuka et al. 2006). Meteor showers are usually associated to comets, and in the case of these asteroids, it is indicative of a past cometary-like activity. The relation between (3200) Phaethon and its associated meteor shower, the Geminds, has been well studied by Gustafson (1989) and Williams \& Wu (1993).

There is also one asteroid, (4015) Wilson-Harrington (also known as comet 107P) that was first discovered as an active comet, then re-discovered as an asteroid (Bowell et al. 1992), and that has never been observed active again. According to Campins \& Swindle (1998), 107P is a potential meteorite producing object.

Meng et al. (2004) noticed that all these "activated asteroids" in the NEA population have spectra and colors compatible with B-type asteroids. Licandro et al. (2007) suggested a link between activated asteroids in the NEA population and MBCs, as their colors and/or spectra correspond to those of $\mathrm{Cb}$ - or B-type asteroids, therefore, the activated asteroids in the NEA population are likely MBCs scattered from the MB to NEA orbits.

In Fig. 4 the spectra of $133 \mathrm{P}$ and (3200) Phaethon (from Licandro et al. 2007) are plotted together. The spectrum of 133P has similarities with the spectrum of (3200) Phaethon: both present neutral to slightly bluish slopes in the 5000-9000 spectral region and a drop of in the UV below $4500 \AA$. Anyway, there are some differences indicative of possible differences in the composition of the surface of both objects: (3200) Phaethon spectrum is slightly bluer $\left(S_{\mathrm{V}}^{\prime}=-4 \% / 1000 \AA\right)$ than $133 \mathrm{P}$ one, but with a slope similar to the spectrum of $176 \mathrm{P}$ $\left(S_{\mathrm{V}}^{\prime}=-3 \% / 1000 \AA\right)$; the UV absorption in the spectrum of (3200) Phaethon is not as well defined as in the spectrum of 

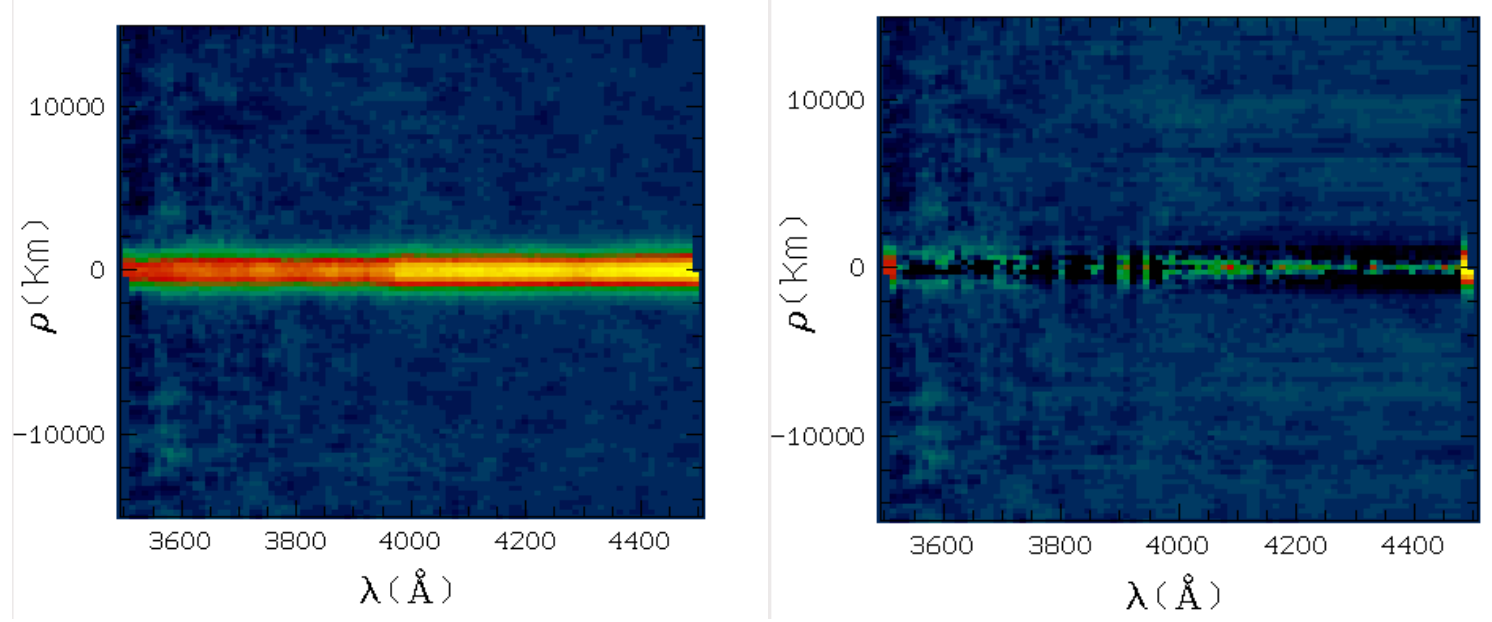

Fig. 5. Combined spectrum of $133 \mathrm{P}$ in the region of the $\mathrm{CN}$ band (left) and that of the "gas" (right), after the subtraction of the dust spectrum. The intensity scale is the same for both and the look up table is logarithmic. The spectral region is between 3500 and $4500 \AA$ and the spatial region is from -15000 to $15000 \mathrm{~km}$ of projected nucleocentric distance $(\rho)$. Notice that no emission bands are seen within the SNR. The region very close to the object photometric center is very noisy because the emission of the dust is strong, but a few thousands km farther the SNR improves.

$133 \mathrm{P}$, in fact $\tau=0.98$, almost 1 in the case of Phaethon, indicative of a shallow absorption in contrast with the $\tau=0.89$ value in the case of $133 \mathrm{P}$.

Licandro et al. (2007) argued that (3200) Phaethon's surface has abundant phyllosilicates and that this object is unlikely to have a cometary origin. The similarity between (3200) Phaethon's spectrum and that of 133P and 176P then also supports an asteroidal nature for these MBCs.

Recent results show that (3200) Phaethon is likely to be a fragment of asteroid (2) Pallas family (de León et al. 2010), in fact, its visible spectrum is identical to those of the Pallas family asteroids. The activity of (3200) Phaethon, 133P and 176P suggest that both, (2) Pallas and (34) Themis, produced fragments with the ability to develop cometary-like activity.

\subsection{MBCs and comets}

To explore the possible cometary origin of 133P, we plot in Fig. 4 the spectrum of 162P/Siding-Spring from Campins et al. (2006), one of the best SNR spectrum of a comet nucleus, rebinned to the same spectral resolution as the other objects presented in this paper. Notice that $162 \mathrm{P}$ 's spectrum is very different to that of the Themis family asteroids and (3200) Phaethon. The spectrum of $162 \mathrm{P}$ is compatible with the spectra of D-type asteroids: featureless with a steep red slope $(>0.07 \% / 1000 \AA)$ and with no absorption feature in the UV. The large majority of comet nuclei with observed spectra have a spectrum similar to that of $162 \mathrm{P}$ and compatible with that of P- or D-type asteroids (Jewitt 2002; Licandro et al. 2002; Campins et al. 2006, 2007; Snodgrass et al. 2008). Also the asteroids in cometary orbits (objects with an asteroidal aspect but with cometary-like orbits) that more likely have a cometary origin (those with $T_{\mathrm{J}}<2.7$ ) are all P- and D-types (Licandro et al. 2008). P- and D-type asteroids have featureless spectra, with a slightly red to very red slope $\left(S_{\mathrm{V}}^{\prime}>2 \% / 1000 \AA\right)$, and it is assumed that they are composed of primitive material. The fact that the MBCs and comets present spectra of different taxonomical types argues also against a cometary origin of the MBCs.

\section{An upper limit for the $\mathrm{CN}$ production rate of 133P/Elst-Pizarro}

Even if, as mentioned in Sect. 2, the spectra of 133P obtained with the VLT are not useful in getting the reflectance, they can be used to look for any signature of gas emission typically observed in cometary comae, that of $\mathrm{CN}\left(\mathrm{B}^{2} \Sigma^{+}-\mathrm{X}^{2} \Sigma^{+} ; \Delta v=0\right)$ at $\sim 3800 \AA$ being the most prominent one. When observed with the VLT, 133P presented a faint tail and a diffuse dust coma, so the detection of any gas emission would be a strong test to determine if the observed tail and coma were produced by ice sublimation as in normal comets.

The spectra of 133P obtained with the VLT were calibrated in intensity (erg/s cm $\mathrm{cm}^{2} \AA$ ster) and in projected distance $(\mathrm{km})$ along the slit, using the spectrum of the spectrophotometric standard. To increase the signal to noise ratio all the calibrated spectra were summed.

A two dimensional cometary dust spectrum was obtained and subtracted from the 133P spectrum. First, the "color" (not the real dust color because the observations were not made in parallactic angle) of the dust was found by comparing the solar analogue (SA) and comet spectra in regions out of possible gas emission. Then the dust profile along the slit was measured in the same regions. The final 2D dust spectrum shown in Fig. 5 is composed by the colored SA spectrum and the profile of the 133P dust. The subtraction gives the cometary gas spectrum shown in Fig. 5. In Fig. 6 we also present the extracted 1D spectrum from a region around $1000 \mathrm{~km}$ from the asteroid and compare it with the spectrum of the solar analogue. From Figs. 5 and 6 we conclude that no emission bands are detected above noise limits. In particular, no gas emission is detected in the $\mathrm{CN}$ band at $3800 \AA$ (see Fig. 7) that shows the "profile" of $\mathrm{CN}$ along the slit.

In order to have an upper limit of the $\mathrm{CN}$ bands intensity, the standard deviation was measured in correspondence with the $\mathrm{CN}$ bands in two regions at projected nucleocentric distance of $3000 \mathrm{~km}$, over a width of $2000 \mathrm{~km}$ by $26 \AA$. The spectrum has the highest SNR in those regions, since the dust contribution is already very low. Each region has a standard 


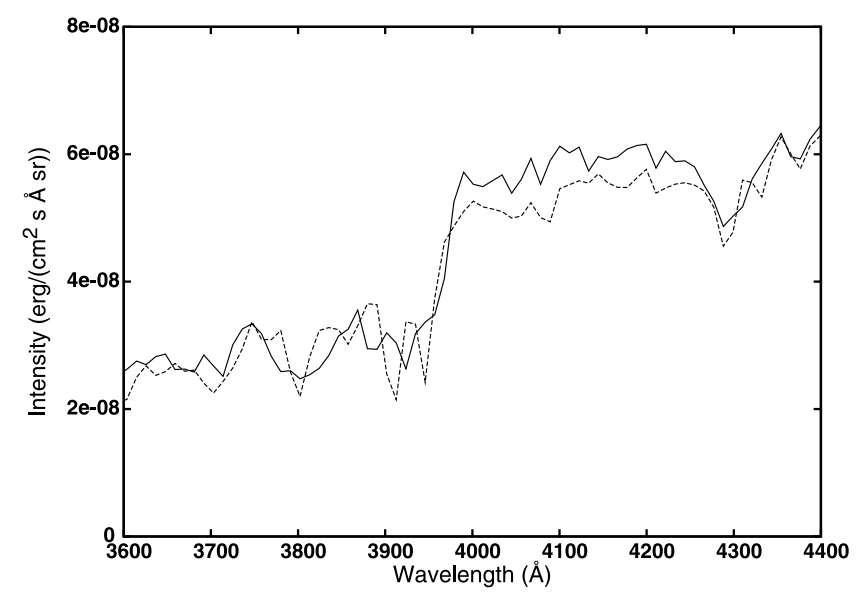

Fig. 6. Section of the inner region $(-1000 \mathrm{~km}<\rho<1000 \mathrm{~km})$ spectrum of 133P (full line), compared with the spectrum of the solar ana$\log$ (dashed line), normalized to 133P spectrum. Notice that there is not obvious gas emission band in the $133 \mathrm{P}$ spectrum.

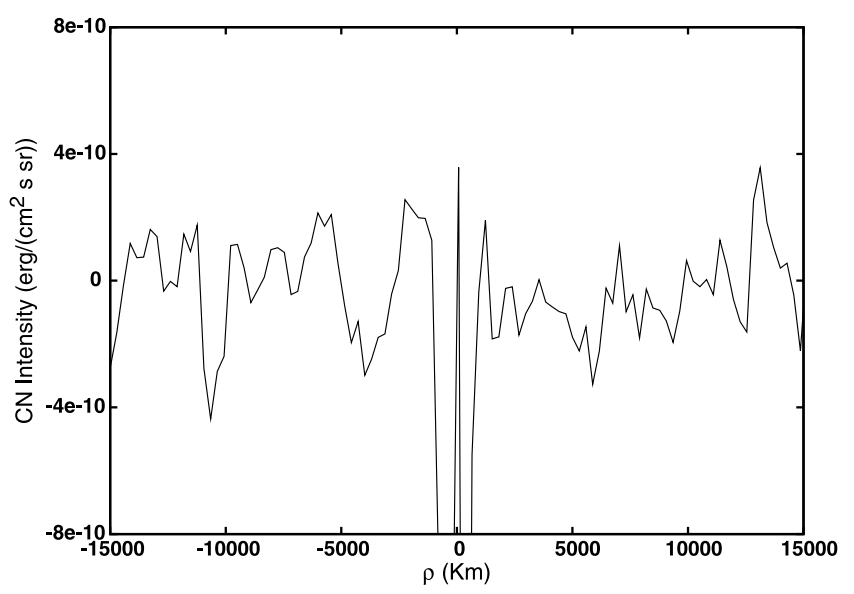

Fig. 7. Profile of the "gas" spectrum in the region covered by the $\mathrm{CN}$ passband in function of the projected nucleocentric distance $\rho$. In the inner region the gas profile is very noisy because the emission of the dust is stronger. Out of the inner region no signature of $\mathrm{CN}$ is seen in the profile within the SNR, the profile is just noise with a $\sigma=6.6 \times$ $10^{-10}$ (erg/ $\mathrm{cm}^{2}$ s ster).

deviation $\sigma=3.6 \times 10^{-11} \mathrm{erg} /\left(\mathrm{cm}^{2}\right.$ ster $\left.\AA \mathrm{s}\right)$, that, considering the $\Delta \lambda=26 \AA$ and the use of 2 regions, gives a total $\sigma=6.6 \times$ $10^{-10} \mathrm{erg} /\left(\mathrm{cm}^{2}\right.$ ster $\left.\mathrm{s}\right)$. Assuming a limit of $3 \sigma$ the upper limit of the intensity is $2 \times 10^{-9} \mathrm{erg} /\left(\mathrm{cm}^{2}\right.$ ster s). With a CN g-factor of $3.9 \times 10^{-14} \mathrm{erg} /(\mathrm{s} \mathrm{mol})$ (Schleicher 2010 and available online asteroid. lowell.edu/comet/comet_intro) the upper limit of the column density is $\approx 7 \times 10^{5} \mathrm{~cm}^{-2}$.

Using the vectorial model (Festou 1981, available online, www.boulder.swri.edu/wvm), the corresponding upper limit of $\mathrm{CN}$ production rate $Q(\mathrm{CN})$ was computed. It is $1.3 \times 10^{21} \mathrm{~mol} / \mathrm{s}$, about three orders of magnitude lower than the $Q(\mathrm{CN})$ determined for Jupiter family comets observed at similar heliocentric distances (A'Hearn et al. 1995). The upper limit of $\mathrm{CN}$ mass loss is $0.6 \times 10^{-4} \mathrm{~kg} / \mathrm{s}$. Assuming a $Q\left(\mathrm{CN} / \mathrm{H}_{2} \mathrm{O}\right) \sim 0.001$ (A'Hearn et al. 1995) gives a water production rate $Q\left(\mathrm{H}_{2} \mathrm{O}\right)=1.5 \times 10^{24} \mathrm{~mol} / \mathrm{s}$, corresponding to a water mass loss $4 \times 10^{-2} \mathrm{~kg} / \mathrm{s}$. In any case, we have to keep in mind that this is an upper limit and that the ratio of $\mathrm{CN}$ over $\mathrm{H}_{2} \mathrm{O}$ production rate may vary with the comet and the heliocentric distance.
Excluding the contribution of the tail, Bagnulo et al. (2010) give a diffuse $A f \rho$ of the dust of the order of $0.6-0.9 \mathrm{~cm}$. Assuming the empiric formula given in (Kidger 2004) a rough approximation of the dust mass loss of the order of $0.7-1.6 \mathrm{~kg} / \mathrm{s}$ can be derived. The $A f \rho / Q(\mathrm{CN})>2-3 \times 10^{-22} \mathrm{~cm} /(\mathrm{mol} \mathrm{s})$ is compatible with the values determined for other Jupiter family comets observed at similar heliocentric distances (A'Hearn et al. 1995).

\section{Discussion and conclusions}

We present the visible spectra of MBCs 133P/Elst-Pizarro and 176P/LINEAR, and of three Themis family asteroids (62) Erato, (379) Huenna and (383) Janina. The spectra of 133P and 176P are very similar to each other and to those of B-type asteroids. The measured spectral slopes in the visible are $S_{\mathrm{V}}^{\prime}=$ $0 \pm 1 \% / 1000 \AA$ and $-3 \pm 1 \% / 1000 \AA$ for $133 \mathrm{P}$ and $176 \mathrm{P}$, respectively.

The spectra of $133 \mathrm{P}$ and $176 \mathrm{P}$ are very similar to those of the three Themis family asteroids, supporting the hypothesis that $133 \mathrm{P}$ and $176 \mathrm{P}$ are Themis family members and unlikely are interlopers that originated in any of the known cometary reservoirs (the Oort Cloud and the TNB).

In order to determine whether water ice sublimation is the mechanism that produces the observed activity of MBCs, the spectra of $133 \mathrm{P}$ obtained with the VLT are used to check the presence of gas emission. In particular, we searched for $\mathrm{CN}$ emission around $0.38 \mu \mathrm{m}$. No $\mathrm{CN}$ emission is detected within the SNR of the data. An upper limit for $\mathrm{CN}$ production rate of $Q(\mathrm{CN})=1.3 \times 10^{21} \mathrm{~mol} / \mathrm{s}$, is determined, about three orders of magnitude lower than the $Q(\mathrm{CN})$ determined for Jupiter family comets observed at similar heliocentric distances (A'Hearn et al. 1995). That means that, if water-ice sublimation is the activation mechanism, the gas production rate is very low and/or the parent molecules of CN present in the nuclei of normal comets are much less abundant in the MBCs.

The spectra of the MBCs presented in this paper are very different from that of comet 162P/Siding-Spring and most other comet nuclei. Considering that most cometary nuclei and asteroids in cometary orbits (likely dormant comets) have spectra and colors typical of P- and D-type asteroids, while MBCs are B-type, we conclude that MBCs and comets are taxonomically different. This argues against a cometary origin of these MBCs.

On the other hand, both MBCs have a spectrum that is very similar to that of the best studied activated asteroids in the nearEarth population, (3200) Phaethon. This supports the hypothesis that "activated asteroids" in the NEA population are scattered MBCs (Meng et al. 2004; Licandro et al. 2007). Notice that (3200) Phaethon's surface composition is not compatible with a cometary origin (Licandro et al. 2007), which also supports the asteroidal nature of these MBCs. If the activity is water-ice driven, these results suggest that there are some "activated asteroids" in the NEA and main belt population that were able to retain water ice that sublimates under certain circumstances.

Additionally, (3200) Phaethon is likely an scattered asteroid from the Pallas family (de León et al. 2010), while 133P and $176 \mathrm{P}$ are Themis family asteroids. Therefore, two large B-type asteroids, (2) Pallas and (24) Themis, are likely parent bodies of asteroids that could have retained some volatiles and present cometary-like activity. Exploring the volatile content of icy minor bodies is critical for understanding the physical conditions and the mechanisms of planetary formation, and also addresses the question of the origin of Earth's water. If the outer main belt 
has a large population of asteroids with ice, they could have contributed to the water on Earth. Finally, this indicates the extent and origin of volatiles in asteroids that could be used as resources for space exploration.

Acknowledgements. This article is based on observations made with the WHT, TNG and NOT telescopes operated on the island of La Palma by the ING, FFG-INAF and NOTSA, respectively, in the Spanish "Observatorio del Roque de los Muchachos", and with the VLT of the European Southern Observatory ESO (program number 279.C-5035). J.L. gratefully acknowledges support from the spanish "Ministerio de Ciencia e Innovación" project AYA2008-06202-C0302. N.P.A. acknowledges support from NASA Postdoctoral Program, administered by Oak Ridge Associated Universities through a contract with NASA. H.C. gratefully acknowledges support from NASA and NSF.

\section{References}

A'Hearn, M. C., Millis, R. L., Schleicher, D. G., Osip, D. J., \& Birch, P. V. 1995, Icarus, 118,223

Bagnulo, S., Tozzi, G. P., Boehnhardt, H., Vincent, J.-B., \& Muinonen, K. 2010, A\&A, 514, A99

Bell, J. 1989, Icarus, 78, 426

Boehnhardt, H., Sekanina, Z., Fiedler, A., et al. 1997, Highlights Astron., 11A, 233

Bottke, W. F., Morbidelli, A., Jedicke, R., et al. 2002, Icarus, 156, 399

Bowell, E., Hapke, B., Domingue, D., et al. 1989, in Asteroids II, ed. R. P. Binzel,

T. Gehrels, \& M. S. Matthews (Univ. of Arizona Press, Tucson), 524

Bowell, E., West, R., Heyer, H., et al. 1992, IAUC, 5585

Bus, S. J. 1999, Ph.D. Thesis, Massachusetts Institute of Technology

Campins, H., \& Swindle, T. 1998, Met. \& Plan. Sci., 33, 1201

Campins, H., Osip, D. J., Rieke, G. H., \& Rieke, M. J. 1995, P\&SS, 43, 733

Campins, H., Ziffer, J., Licandro, J., et al. 2006, AJ, 132, 1346

Campins, H., Licandro, J., Pinilla-Alonso, N., et al. 2007, AJ, 134, 1626

Campins, H., Kelley, M. S., Fernández, Y., Licandro, J., \& Hargrove, K. 2009, EM\&P, 105, 159

Campins, H., Hargrove, K., Pinilla-Alonso, N., et al. 2010, Nature, 464, 1320

Carusi, A., \& Valsecchi, G. 1982, A\&A, 115, 327

Chamberlin, A. B., McFadden, L.-A., Schulz, R., Schleicher, D. G., \& Bus, S. J. 1996, Icarus, 119, 173

Clark, B., Ziffer, J., Nesvorny, D., et al. 2010, JGRE, 115, E06005

Cunningham, L. E. 1950, IAU Circ., 1250, 3

Delbó, M. 2004. Ph.D. Thesis, Freie Universität, Berlin

Delbó, M., Harris, A. W., Binzel, R. P., Pravec, P., \& Davies, J. K. 2003, Icarus, 166,116

Delbó, M., dell'Oro, A., Harris, A. W., Mottola, S., \& Müller, M. 2007, Icarus, 190, 236

de León, J., Campins, H., Tsiganis, K., Morbidelli, A., \& Licandro, J. 2010, A\&A, 513, A26

de León, J., Licandro, J., Serra-Ricart, M., Pinilla-Alonso, N., \& Campins, H. 2011, A\&A, 517, A23

Drake, M. J., \& Campins, H. 2006, In Asteroids, Comets and Meteorites (Cambridge Univ. Press), 381

Elst, E., Pizarro, O., Pollas, C., et al. 1996, IAUC, 6456

Emery, J. P., Lim, L. F., Marchis, F., \& Cruikshank, D. P. 2008, LPI Contributions, 1405, 8345

Fernández, Y., McFadden, L., Lisse, C., Helin, E., \& Chamberlin, A. 1997, Icarus, 128,114

Fernández, J., Gallardo, T., \& Brunini, A. 2002, Icarus, 159, 358

Fernández, Y., Jewitt, D. C., \& Sheppard, S. S. 2005, AJ, 130, 308

Fernández, Y. R., Kelley, M. S., Lamy, P. L., et al. 2008, LPI Contributions, 1405, 8307
Festou, M. C. 1981, A\&A, 95, 69

Florczak, M., Lazzaro, D., Mothé-Diniz, T., Angeli, C., \& Betzler, A. 1999, A\&ASS, 134, 463

Gustafson, B. 1989, A\&A, 225, 533

Groussin, O., Lamy, P., Toth, I., et al. 2009, Icarus, 199, 568

Haghighipour, N. 2009, M\&PS, 44, 1863

Harris, A. W. 1998, Icarus, 131, 291

Hsieh, H., \& Jewitt, D. 2006, Science, 312, 561

Hsieh, H., Jewitt, D., \& Fernández, Y. 2004, AJ, 127, 299

Hsieh, H., Jewitt, D., \& Fernández, Y. 2009, ApJ, 694, 111

Hsieh, H., Jewitt, D., Lacerda, P., Lowry, S., \& Snodgrass, C. 2010, MNRAS, 403,363

Ivezic, Z., Lupton, R. H., Jurić, M., et al. 2002, AJ, 124, 2943

Jakosky, B. M. 1986, Icarus, 66, 117

Jewitt, D. 2002, AJ, 123, 1039

Kidger, M. R. 2004, A\&A, 420, 389

Keihm, S. J. 1984, Icarus, 60, 568

Kosai, H. 1992, Celest. Mech. Dyn. Astron., 54, 237

Kraemer, K. E., Lisse, C. M., Price, S. D., et al. 2005, AJ, 130, 2363

Kresak, L. 1982, Bull. Astron. Inst. Czech., 33, 104

Kührt, E., \& Giese, B. 1989, Icarus, 81, 102

Lamy, P., \& Toth, I. 2009, Icarus, 201, 674

Landolt, A. 1992, AJ, 104, 340

Levison, H. F., Terrell, D., Wiegert, P. A., Dones, L., \& Duncan, M. J. 2006, Icarus, 182, 161

Levison, H., Bottke, W., Gounelle, M., et al. 2009, Nature, 460, 364

Licandro, J., Campins, H., Hergenrother, C., \& Lara, L. M. 2002, A\&A, 398 L45

Licandro, J., Campins, H., Mothé-Diniz, T., Pinilla-Alonso, N., \& de León, J. 2007, A\&A, 461, 751

Licandro, J., Alvarez-Candal, A., de León, J., et al. 2008, A\&A, 481, 861

Makovoz, D., \& Khan, I. 2005, Astronomical Data Analysis Software and Systems XIV, 347, 81

Marzari, F., Farinella, P., \& Vanzani, V. 1995, A\&A, 299, 267

Meng, H., Zhu, J., Gong, X., et al. 2004, Icarus, 169, 385

Mothe-Diniz, T., Roig, F., \& Carvano, J. M. 2005, Icarus, 174, 54

Mottl, M., Glazer, B., Kaiser, R., \& Meech, K. 2007, Chemie der Erde Geochemistry, 67, 253

Mueller, M. 2007. Surface Properties of Asteroids from Mid-Infrared Observations and Thermophysical Modeling, Digitale Dissertation, Freie Universitaet Berlin, available from: http://www.diss.fu-berlin.de/ $2007 / 471 /$ indexe.html

Müller, T. G., \& Lagerros, J. S. V. 1998, A\&A, 338, 340

Nesvorny, D., Bottke, W., Vokrouhlicky, D., et al. 2008, ApJ, 679, L143

Ohtsuka, K., Sekiguchi, T., Kinoshita, D., et al. 2006, A\&A, 450, 25

Osip, D., Campins, H., \& Schleicher, D. G. 1995, Icarus, 114, 423

Reach, W. T. Kelley, M. S., \& Sykes, M. V. 2007, Icarus, 191, 298

Rivkin, A. S., \& Emery, J. 2010, Nature, 464, 1322

Rousselot, P., Dumas, C., \& Merlin, F. 2011, Icarus, 211, 553

Schleicher, D. G. 2010, AJ, 140, 973

Schorghofer, N. 2008, ApJ, 682, 697

Snodgrass, C., Lowry, S. C., \& Fitzsimmons, A. 2008, MNRAS, 385, 737

Spencer, J. R. 1990, Icarus, 83, 27

Tanga, P., Cellino, A., Michel, O., et al. 1999, Icarus, 141, 65

Tedesco, E. F., Noah, P., Noah, M., \& Price, S. 2004. IRAS Minor Planet Survey, IRAS-A-FPA-3-RDR-IMPS-V6.0, NASA Planetary Data System

Tholen, D. J. 1989, in Asteroids II, ed. R. P. Binzel, T. Gehrels, \& M. S. Matthews (Tucson: Univ. of Arizona Press), 298

Toth, I. 2006, A\&A, 446, 333

Whipple, F. 1983, IAUC, 3881

Williams, I. P., \& Wu, Z. D. 1993, MNRAS, 264, 659

Zappalà, V., Cellino, A., Farinella, P., \& Knezevic, Z. 1990, AJ, 100, 2030 